\title{
Highly sensitive and selective biosensor based on graphene oxide coated long period grating
}

M. S. Shaikh

C. Liu

M. Partridge

S. W. James

W. D. Zhu

X. Chen 


\title{
Highly sensitive and selective biosensor based on graphene oxide coated long period grating
}

\author{
M. S. Shaikh ${ }^{\mathrm{a}}$, C. Liu ${ }^{\mathrm{a}}$, M. Partridge ${ }^{\mathrm{b}}$, S. W. James ${ }^{\mathrm{b}}$, W. D. Zhu ${ }^{\mathrm{c}}$, X. Chen ${ }^{\mathrm{a}, *}$ \\ ${ }^{a}$ School of Electronic Engineering, Bangor University, LL57 1UT, United Kingdom \\ ${ }^{\mathrm{b}}$ Centre for Engineering Photonics, Cranfield University, MK43 0AL, United Kingdom \\ ${ }^{\mathrm{c}}$ Department of Applied Science and Technology, Saint Peter's University, NJ 07306, USA
}

\begin{abstract}
We propose an optical fiber immunosensor based on graphene oxide coated dual-peak long period grating (GO-dLPG), in which GO-IgG linking layer is used for rapid immunoassays. The binding interaction between antibody and antigen produced a detectable optical signal in terms of grating resonant wavelength shift, which was proportional to the analyte concentration. By deposition of GO overlay, the bulk RI sensitivity of dLPG was enhanced around $150 \%$. The GOcoated dLPG was biofunctionalized by the immobilization of IgG to generate the biosensor. The IgG-bound GO-dLPG was used to detect the anti-IgG and anti-PSA, respectively, demonstrating high sensitivity and selectivity. The GO-dLPG biosensor can be further developed as a biosensing platform with advantages of label-free, real-time and low limit of detection.
\end{abstract}

Keywords: Graphene oxide; Optical fiber biosensor; Long period grating; Label-free; Antibody-Antigen

\section{INTRODUCTION}

Biosensors have become a major field of research due to the large number of possible applications. As an analytical device, a biosensor consists of two major components - a molecular recognition element which detects a target analyte and a transducer which demonstrates the interaction between the recognition element and the analyte ${ }^{1}$. The recognition element converts the biochemical information of the target analyte, e.g. concentration, into a suitable chemical or physical output signal which has a defined sensitivity. The transducer converts the output of the recognition element into electrical or optical signals which can be easily detected, monitored and measured.

Over the last few decades, fiber grating sensor technology has been extensively developed due to the fiber's inherent merits of compact size, light weight, immunity to electromagnetic interference as well as the advantages of real-time, multiplexing, and in-line monitoring. Various fiber optic chemical and biochemical sensors have been proposed through the use of fiber Bragg gratings (FBGs), long period gratings (LPGs), and tilted fiber gratings (TFGs) ${ }^{2-8}$. These sensors operate as the refractive index (RI) based sensor, the chemical or biological events induce a RI change resulting a quantifiable optical signal, which can be monitored through grating resonance change. It has been reported that the maximum RI sensitivity of the LPG can be achieved when the surrounding refractive index (SRI) approaches the value of the fiber cladding ${ }^{6,7}$. Moreover, the grating based biosensor can demonstrate better performance and selectivity by depositing a thin functional coating layer over the grating region ${ }^{9-11}$.

Graphene, as a new member of carbon family, was first introduced as two-dimensional structure in $2004{ }^{12}$. Graphene has a single layer of $\mathrm{sp}^{2}$ carbon atoms ordered in a two-dimensional honeycomb lattice and it is the basic building block of 0D, 1D and 3D carbon allotropes. It owns superior electrical, mechanical, optical, and electrochemical properties and can also be chemically functionalized. As the derivative of graphene, graphene oxide (GO) contains both $s p^{2}$ - and $s p^{3}$ hybridized carbon atoms as well as different oxygen-containing functional groups, which can be used for immobilization of biomolecules ${ }^{13}$. The excellent capabilities in biocompatibility, solubility and selectivity make GO a very promising carbon-based nanomaterial for widespread biosensing applications.

*x.chen@bangor.ac.uk; phone 441248 382480; fax 441248361429

Optical Fibers and Sensors for Medical Diagnostics and Treatment Applications XVII, edited by Israel Gannot, Proc. of SPIE Vol. 10058, 100580G · (C) 2017 SPIE · CCC code: 1605-7422/17/\$18 · doi: 10.1117/12.2252371 
In this work, we report the implementation of an immunosensor based on GO-coated dual-peak LPG (GO-dLPG) for real time monitoring of the kinetic binding interaction of antibody and antigen. The GO-coated dLPG demonstrated extremely high RI sensitivity in the low RI region relevant to biological assays and events. IgG was immobilized on GO overlay via EDC/NHS scheme. The GO-IgG linking layer offered an analytical platform for the detection of immunosensing between IgG and specific anti-IgG.

\section{DLPG FABRICATION, GO DEPOSITION, RI SENSITIVITY}

\subsection{Inscription of dual-peak LPG}

In this work, the grating with a period of $109 \mu \mathrm{m}$ and length of $4 \mathrm{~cm}$ was used. The dLPGs were fabricated by a pointby-point overwrite method with a Nd:YAG laser operating at $266 \mathrm{~nm}^{14}$. The inscription laser beam was conditioned by using a combination system of a focusing lens and a slit, which were all computer controlled. The optical fiber used in these experiments was PS750 Boron doped photosensitive fiber (Fibercore) with a cut off wavelength of $620 \mathrm{~nm}$. The fiber was mounted on a translation stage with its axis of movement oriented orthogonally to the laser beam. The grating transmission spectrum was monitored during the inscription process to control the laser exposure with the desirable dualpeak feature. As has been previously reported ${ }^{7,8}$, a set of dispersion-turning-points (DTP) exists in phase-matching curves resulting in the conjugate cladding modes observed as dual-peak features in the transmission spectrum. For a 109 $\mu \mathrm{m}$-period dLPG, the light coupling from core mode to high order cladding modes leads to two attenuation peaks, at $804.5 \mathrm{~nm}$ (blue peak) and $881.0 \mathrm{~nm}$ (red peak), in the transmission spectrum measured in air (black line, Fig. 4a) with respect to the same conjugate cladding mode. Deal peaks will shift to opposite sign to the same external RI perturbation.

\subsection{Deposition of GO on fiber device surface}

Deposition of GO on device surface could enhance the performance of the sensor. In this work, the deposition method has been developed to coat the GO layer over the optical fiber surface.
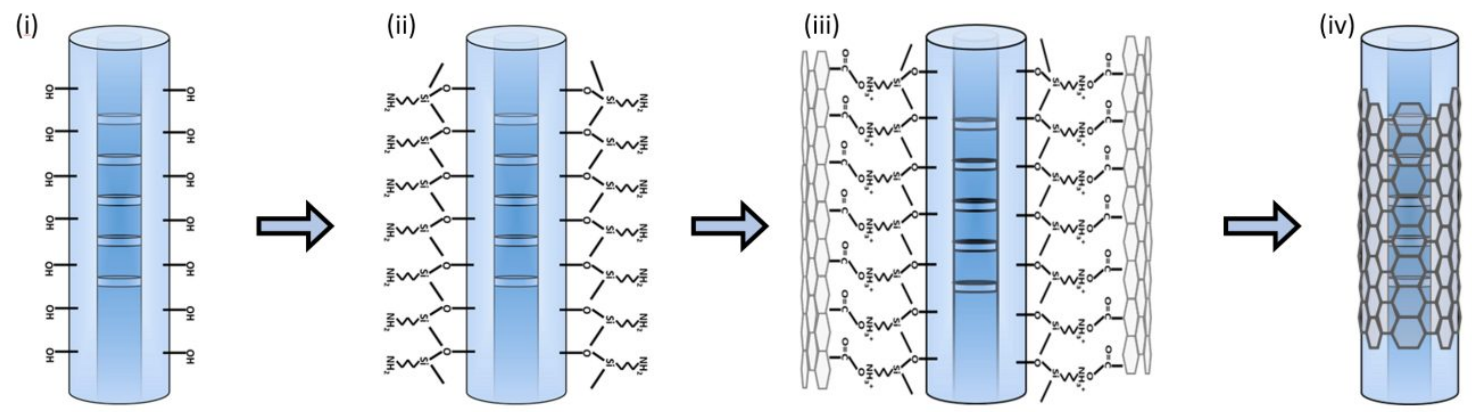

Figure 1. Schematic of the GO deposition on fiber optic sensor surface.

As the schematic in Fig.1, (i) The fiber was cleaned by immersion in acetone for $10 \mathrm{~min}$ and by immersion in HCL (1 M) for $10 \mathrm{~min}$ at room temperature to remove the organic contaminant, rinsed with DI water thoroughly and dried. Then the fiber was immersed in $1 \mathrm{M} \mathrm{NaOH}$ solution for $1 \mathrm{~h}$ to enrich the number of silanol groups on the fiber surface. (ii) The fiber was immersed in a 5\% (3-Aminopropyl)triethoxysilane (APTES) solution for $2 \mathrm{~h}$. The APTES reacted with the hydroxyl groups on the silica surface which resulted in the formation of Si-O-Si bonds. The fiber was washed with ethanol and placed on a hot plate at $50{ }^{\circ} \mathrm{C}$ for $30 \mathrm{~min}$. (iii) Finally, the APTES-silanized fiber was incubated into 0.06 $\mathrm{mg} / \mathrm{mL}$ GO aqueous dispersion contained in a mini-bath placed on a hot plate at $50^{\circ} \mathrm{C}$. When a coated layer was observed on the surface, the fiber was removed from the GO dispersion and dried in air to give a thin layer of GO (iv).

\subsection{Refractive index properties of bare and GO-coated dLPG}

It was reported that the dual-peak cladding modes are extremely sensitive to SRI change since they are in close proximity to DTP. The RI sensitivities of bare and GO-coated dLPGs were investigated by applying fiber sensors with a set of aqueous sucrose solutions. The sucrose solutions with concentrations of $0,10,20,30,35,40,45,50,55$ and $60 \%$ were prepared with measured RIs of $1.332,1.347,1.362,1.381,1.390,1.399,1.408,1.419,1.430$ and 1.441, respectively. Fig. 2a plots the bare dLPG transmission spectra under different sucrose concentrations. As expected the 
dual peaks move to opposite directions when the SRI increased. Fig $2 b$ shows the dependence of dual-peak wavelength shift against the SRI for bare dLPG (open symbols) and GO-dLPG (solid symbols), both demonstrating a non-linear relationship. The bare dLPG achieved the RI sensitivities of $233.1 \mathrm{~nm} / \mathrm{RIU}$ and $2034.4 \mathrm{~nm} / \mathrm{RIU}$ for low RI (1.332-1.347) and high RI (1.419-1.441) regions, respectively. For the GO-coated dLPG, the RI sensitivities were $349.5 \mathrm{~nm} / \mathrm{RIU}$ and $2983.5 \mathrm{~nm} / \mathrm{RIU}$ for the corresponding low and high RI regions. With the GO deposition, the RI sensitivities of dLPG sensor have been increased around $150 \%$. The RI sensing mechanism relies on the sensitivity of LPG resonance to the properties of coating material. The GO overlay with high RI enhanced the light-matter interaction between cladding and surrounding-medium leading to the increase in RI sensitivity.

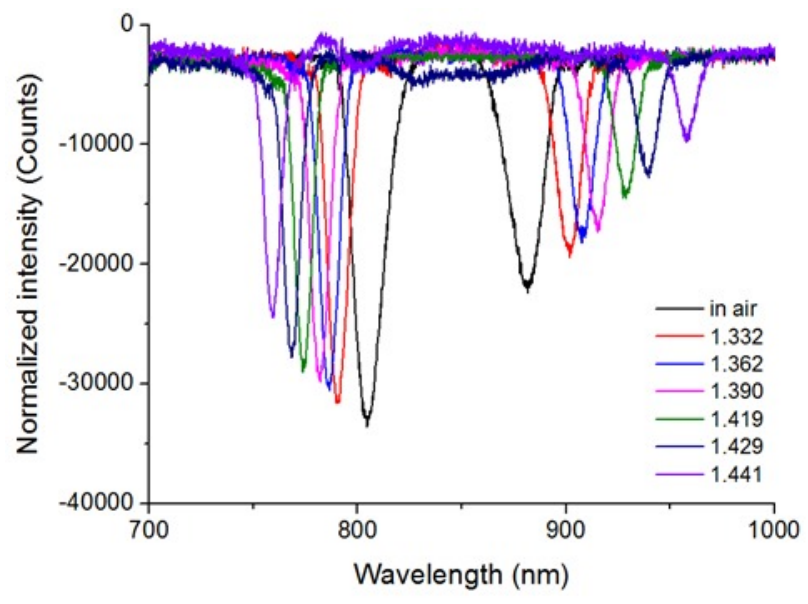

(a)

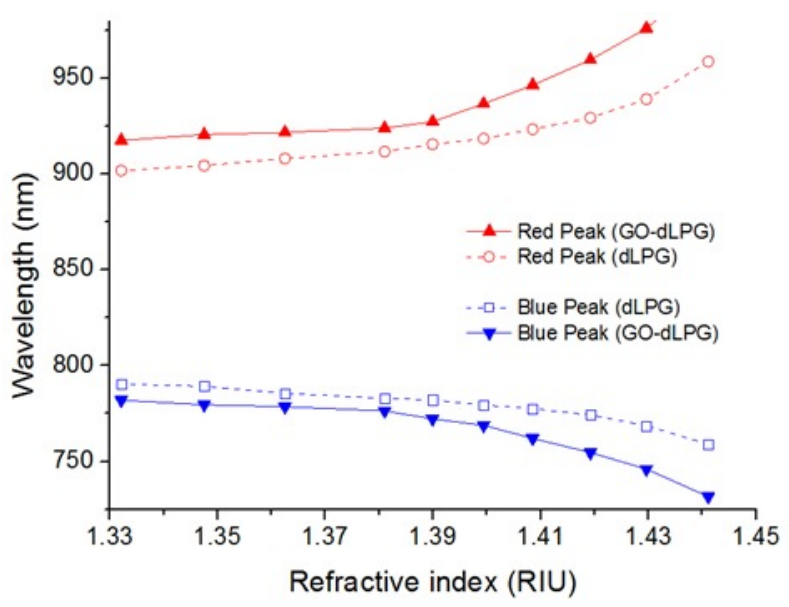

(b)

Figure 2. (a) Spectra evolution of dLPG at different RI (b) dLPG wavelength shift against RI.

\section{BIOFUNCTIONALIZATION AND LABEL-FREE IMMUNOSENSING}

Immobilization of biomolecules on the sensor surface is an important step in the biosensor generation. In this work, $\operatorname{IgG}$ was immobilized on the GO surface by the use of heterobifunctional cross-linkers of EDC/NHS ${ }^{15}$.

The GO-dLPG was immersed into the mixture of EDC $(20 \mathrm{mM})$ and NHS $(40 \mathrm{mM})$ solution in a Teflon bath device for $1 \mathrm{~h}$ at room temperature. The cross-linker of EDC/NHS activated the terminal carboxyl group (-COOH) on the GO surface for further immobilization of antibody ${ }^{12}$. After washing with $1 \times$ PBS buffer, the fiber device was immersed in 1 $\mathrm{mg} / \mathrm{mL}$ rabbit IgG solution for $2 \mathrm{~h}$. The fiber device was then washed with $1 \times \mathrm{PBS}$ for several times to ensure the removal of any non-bound antibodies on the GO surface. The unreacted sites on the GO surface were passivated by immersing the IgG-immobilized GO-dLPG into $1 \%$ BSA solution for $30 \mathrm{~min}$. Here, the antibody-immobilized GO-dLPG was ready as a biosensor for antibody/antigen immunosensing detection.

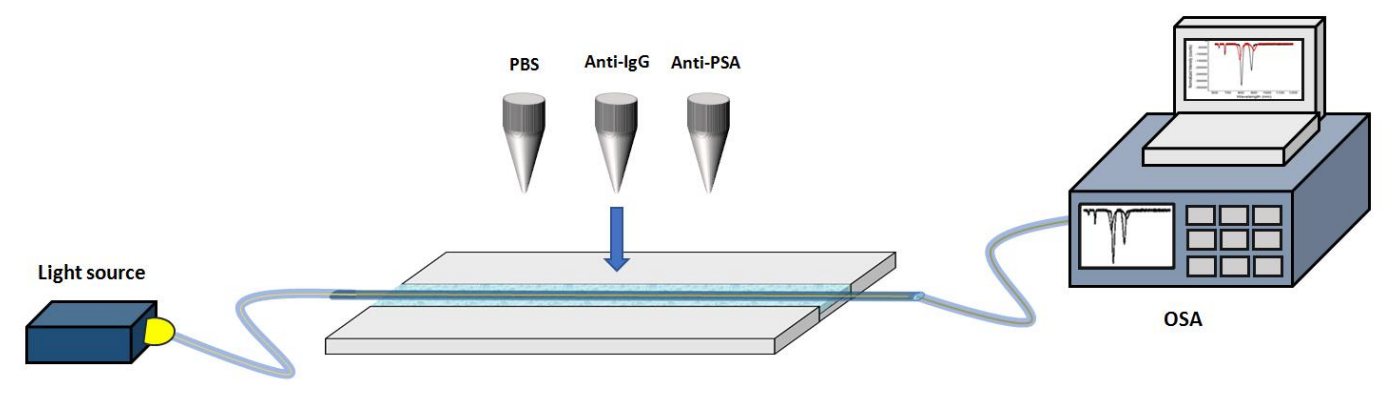

Figure 3. Schematic of experimental setup for biosensing detection. 
The setup for biosensing experiment is shown in Fig. 3. The Tungsten Halogen Lamp was used as the light source along with an Optical Spectrum Analyser (OSA) for monitoring the transmission spectrum of the GO-dLPG. The IgGimmobilized GO-dLPG was placed into a V-groove and kept straight by both ends fixed and the biosensing detection was carried out at controlled temperature of $22.1{ }^{\circ} \mathrm{C}$ to avoid the cross-effect from strain and temperature.

The GO-dLPG based biosensor for specific immunosensing was demonstrated by the detections of anti-IgG and antiPSA. The red peak of GO-dLPG was used to monitor the wavelength shift during the immunoassays and its movement is plotted in Fig. 4a. The IgG-bound GO-dLPG was immersed in PBS buffer for 5 min providing a stable baseline over which the grating peak was monitored. The kinetic bioaffinity binding between IgG-immobilized GO-dLPG and target goat anti-rabbit $\operatorname{IgG}$ with concentration of $0.1 \mu \mathrm{g} / \mathrm{mL}$ was evaluated by monitoring grating resonant wavelength shift in real-time. As shown in Fig. 4a, it may be regarded that there are two stages associated with the bioaffinity binding interaction. The rapid binding interaction stage occurred in the first three minutes showing a wavelength shift rate of 200 $\mathrm{pm} / \mathrm{min}$, then a $27-\mathrm{min}$ steady stage followed when the signal achieved saturation gradually. The overall wavelength shift induced by the immunoassay process is $\sim 760 \mathrm{pm}$. In comparison with the core-etched FBG based biosensor ${ }^{5}$, the GOdLPG based biosensor has achieved around seven times higher reaction rate.

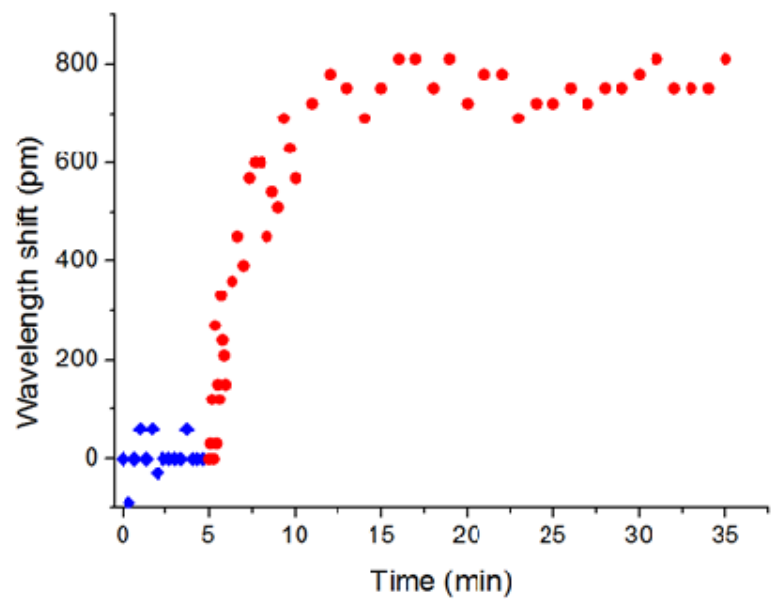

(a)

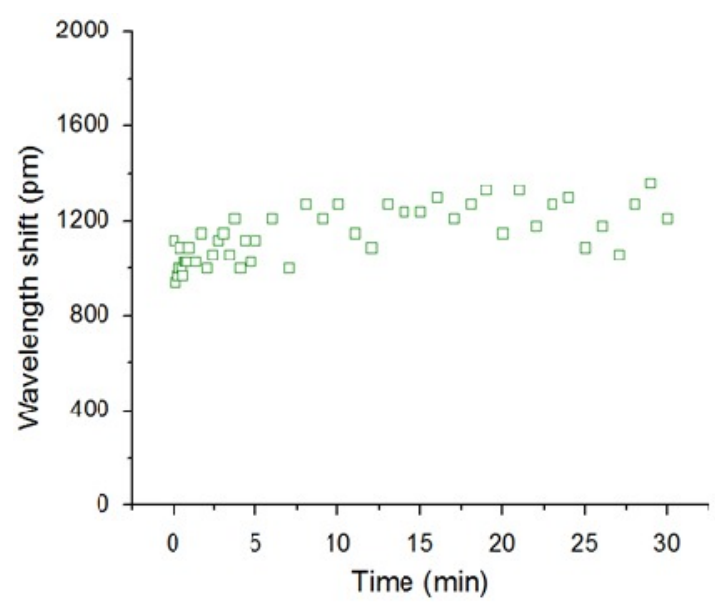

(b)

Figure 4. Wavelength shift against time during (a) IgG and anti-IgG kinetic binding process and (b) IgG and anti-PSA process.

The specificity of the GO-dLPG biosensor was evaluated by the non-specific reaction between IgG and anti-ProstateSpecific Antigen (anti-PSA). After rinsing with PBS buffer thoroughly, the IgG-bound device was immersed in $1 \mu \mathrm{g} / \mathrm{mL}$ anti-PSA solution for 30 min and the wavelength shifts were plotted in Fig. 4b. In comparison with the IgG/anti-IgG binding process, the response of IgG/anti-PSA process shows non-specific reaction over 30-min observation period. There was an inconspicuous and a step wavelength shift around $150 \mathrm{pm}$ in the very beginning which might be caused by the bulk RI difference between buffer PBS and anti-PSA solution.

\section{CONCLUSION}

We report a biosensor based on GO coated dLPG in this work. The GO layer was successfully deposited on the fiber device surface, resulting in an increase of $\sim 150 \%$ in RI sensitivity. The GO-dLPG was biofunctionalized by immobilization of IgG on GO surface and subsequently used to detect the kinetic binding interaction between IgG and anti-IgG, demonstrating the initial binding rate of $200 \mathrm{pm} / \mathrm{min}$, seven times higher than that of core-etched FBG biosensor. The specificity of the biosensor was implemented by the non-specific reaction between IgG and anti-PSA. Moreover, the working wavelength of 750-950 nm in this work provides a potential 800nm-interrogation system with the advantages of compact size, portable, fast detection speed and low cost. The future work will be continued to detect the target anti-IgG for different concentrations. 


\section{ACKNOWLEDGEMENTS}

The authors acknowledge the support from the EU FP7 PIRSES-2013-612267 (iPhoto-Bio), the Engineering and Physical Sciences Research Council (EPSRC) UK via grant EP/L010437, and Sêr Cymru NRN097.

\section{REFERENCES}

[1] R. Nutiu, L. P.Billen and Y. Li, "Fluorescence-signaling nucleic acid based sensors," Nucleic Acid Switches and Sensors, Springer, $1^{\text {st }}$ ed., pp. 49-72, 2006.

[2] X. D. Wang and O. S. Wolfbeis, "Fiber-optic chemical sensors and biosensors (2008-2012)," Anal Chem. 85(2), 487-508, 2013

[3] J. Canning, " Properties of specialist fibres and Bragg gratings for optical fibre sensors," J of Sensors 2009, 871580,17 pages, 2009

[4] A. Vengsarkar, P. Lemaire, J. Judkins, V. Bhatia, T. Erdogan, and J. Sipe, "Long-period fiber gratings as bandrejection filters," J. Lightwave Technol. 14, 58-64, 1996.

[5] A. N. Chryssis, S. S. Saini, S. M. Lee, H. Yi, W. E. Bentley, and M. Dagenais, "Detecting hybridization of DNA by highly sensitive evanescent field etched core fiber Bragg grating sensors," IEEE J. Sel. Top. Quantum Electron. 11, 864, 2005.

[6] S. W. James and R. P. Tatam, "Optical fiber long-period gratings: characteristics and applications," Meas. Sci. Technology, 14(5), R49-R61, 2003.

[7] X. Shu, L. Zhang, I. Bennion, "Sensitivity characteristics of long-period fiber gratings," J. Lightw. Technol. 20, 255-266, 2002.

[8] X. Chen, L. Zhang, K. Zhou, E. Davies, K. Sugden, I. Bennion, M. Hughes, A. Hine, "Real-time detection of DNA interactions with long-period fiber-grating-based biosensor," Opt. Lett. 32, 2541-2543, 2007.

[9] N. D. Rees, S. W. James, R. P. Tatam and G. J. Ashwell, Optical fiber long-period gratings with LangmuirBlodgett thin-film overlays, Opt. lett. 27(9), 686-688, 2002.

[10] S. Lepinay, A. Staff, A. Ianoul, J. Albert, "Improved detection limits of protein optical fiber biosensors coated with gold nanoparticles," Biosens. Bioelectron. 52, 337-344, 2014.

[11] A. Deep, U. Tiwari, P. Kumar, V. Mishra, S. Jain, N. Singh, P. Kapur, L. Bharadwaj, "Immobilization of enzyme on long period grating fibers for sensitive glucose detection," Biosens Bioelectron. 33, 190-195, 2012.

[12] K.S. Novoselov, A.K. Geim, S.V. Morozov, D. Jiang, Y. Zhang, S.V. Dubonos, I.V. Grigorieva, A. A. Firsov, "Electric field effect in atomically thin carbon films," Science 306 (5696), 666-669, 2004.

[13] E. Morales-Narváez and A. Merkoç, "Graphene Oxide as an Optical Biosensing Platform." Adv. Mater. 24(25), 3298-3308, 2012.

[14] M. Partridge, S. W. James, J. Barrington, R. P. Tatam, "Overwrite fabrication and tuning of long period gratings," Opt. Express 24, 22345-22356, 2016.

[15] C. K. Dixit, S. K. Vashist, B. D. MacCraith, R. O'Kennedy, "Multisubstrate-compatible ELISA procedures for rapid and high-sensitivity immunoassays," Nat. Protoc. 6, 439-445, 2011. 
2017-02-28

\section{Highly sensitive and selective biosensor based on graphene oxide coated long period grating}

\section{Partridge, Matthew}

SPIE

Shaikh MS, Liu C, Partridge M, James SW, Zhu WD, Chena X, Highly sensitive and selective biosensor based on graphene oxide coated long period grating, Proceedings of Optical Fibers and Sensors for Medical Diagnostics and Treatment Applications XVII, 28 - 29 January 2017, San Francisco, California, USA, Paper number 100580G

http://dx.doi.org/10.1117/12.2252371

Downloaded from Cranfield Library Services E-Repository 